\title{
BMJ Open Long-term unmet needs after stroke: systematic review of evidence from survey studies
}

\author{
Ting Chen, ${ }^{1}$ Bo Zhang, ${ }^{1}$ Yan Deng, ${ }^{1}$ Jing-Chun Fan, ${ }^{2}$ Liansheng Zhang, \\ Fujian Song ${ }^{3}$
}

To cite: Chen T, Zhang B, Deng $\mathrm{Y}$, et al. Long-term unmet needs after stroke: systematic review of evidence from survey studies. BMJ Open 2019;9:e028137. doi:10.1136/ bmjopen-2018-028137

- Prepublication history and additional material for this paper are available online. To view these files, please visit the journal online (http://dx.doi. org/10.1136/bmjopen-2018028137).

Received 23 November 2018 Revised 13 February 2019 Accepted 14 March 2019

A) Check for updates

(C) Author(s) (or their employer(s)) 2019. Re-use permitted under CC BY-NC. No commercial re-use. See rights and permissions. Published by BMJ.

${ }^{1}$ School of Public Health, Medical College, Wuhan University of Science and Technology, Wuhan, China ${ }^{2}$ School of Public Health, Gansu University of Traditional Chinese Medicine, Lanzhou, China

${ }^{3}$ Department of Public Health and Epidemiology, Norwich Medical School, University of East Anglia, Norwich, UK

Correspondence to

Dr Fujian Song;

fujian.song@uea.ac.uk

\section{ABSTRACT}

Objectives To synthesise evidence on longer term unmet needs perceived by stroke survivors, and psychometric properties of the tools used to evaluate unmet care needs after stroke.

Design Systematic review.

Setting Community or patients' home.

Participants Stroke survivors.

Methods We searched PubMed, PsycINFO, CINAHL, EMBASE from inception to 31 March 2018 to identify survey studies that evaluated unmet needs perceived by stroke survivors after hospital discharge. Reported unmet needs were categorised under three domains: body functioning, activity/participation and environmental factors. Ranges of prevalence rates of unmet needs reported in studies were presented.

Results We included 19 eligible studies, with considerable heterogeneity in patients, survey methods and results. Psychometric properties of two stroke-specific tools were formally evaluated, indicating their moderate reliability and content/concurrent validity. The median number of reported unmet needs per stroke survivor was from two to five, and the proportion of stroke survivors with at least one unmet needs was on average $73.8 \%$ (range 19.8\%$91.7 \%)$. Unmet needs perceived by stroke survivors included 55 records of unmet body functioning needs, 47 records of unmet activities/participatory needs and 101 records of unmet environmental needs. Common unmet service needs were unmet information needs $(3.1 \%-$ $65.0 \%)$, transport $(5.4 \%-53.0 \%)$, home help/personal care $(4.7 \%-39.3 \%)$ and therapy $(2.0 \%-35.7 \%)$.

Conclusions The prevalence of unmet long-term needs is high among stroke survivors, and there is considerable heterogeneity in type and frequency of specific unmet needs. More research is required to link regular assessment of long-term unmet needs of stroke survivors with the provision of cost-effective patient-centred health and social care services.

\section{INTRODUCTION}

As one of the leading causes of mortality and disability globally, the fatal mortality of patients with acute stroke has been reduced in many countries, and increasingly more stroke survivors are living with disability after discharge from hospital. ${ }^{1}$ There are numerous tools for assessing clinical outcomes after stroke,

\section{Strengths and limitations of this study}

- For patient-centred health and social care, it is important to understand specific needs perceived by stroke survivors.

- This is the first systematic review attempt to synthesis evidence from survey studies of long-term unmet needs perceived by stroke survivors, and to examine psychometric properties of relevant assessment tools.

- Due to considerable heterogeneity, formal meta-analysis could not be conducted.

- Focused on 'unmet needs' and excluded studies that assessed needs after stroke but did not explicitly report results regarding 'unmet needs'.

- Considered unmet needs perceived by stroke survivors only, and excluded studies on unmet needs of informal carers.

such as the Stroke Impact Scale, the Frenchay Activities Index, Homesat and so on. ${ }^{2}$ For patient-centred health and social care, it is important to understand specific needs perceived by stroke survivors. ${ }^{2}{ }^{3}$ However, needs after stroke may be defined differently, and it is often difficult to distinguish needs and other related factors such as patient satisfaction, preferences, health-related quality of life, disability severity, physical and psychological functions. ${ }^{4}$ One practical approach is to define needs as 'the capacity to benefit from healthcare', ${ }^{5}$ which has been criticised for being too restrictive without considering other types of genuine health needs. ${ }^{4}$ Longterm needs of stroke survivors include needs for healthcare services, and needs for social care and other supports. ${ }^{3}$ In addition, needs perceived by patients with stroke may be different from those perceived by healthcare professionals or caregivers.

To inform the provision of health and social care services, it is crucial to identify unmet needs after stroke. ${ }^{2}$ Unmet needs perceived by stroke survivors may be practically evaluated according to patients' perception whether 
they have received any or sufficient help regarding a specific difficulty, or whether a problem has not been addressed at all or sufficiently. ${ }^{6-8}$ Well-conducted survey studies provide quantitative estimates of unmet needs after stroke to inform health and social care policies. Existing systematic reviews focused mainly on specific types of unmet needs or that perceived by carers. ${ }^{9} 10$ Furthermore, there was no systematic assessment of tools used to evaluate unmet needs after stroke. These systematic reviews aimed to evaluate types and rates of long-term unmet needs after stroke (LUNS) for making health and social care policies, and to understand what tools could be used for assessing long-term unmet needs. Therefore, we synthesised evidence from survey studies that evaluated long-term unmet needs perceived by stroke survivors, and examined psychometric properties of the tools for assessing unmet care needs of postacute stroke survivors.

\section{METHODS}

The review protocol is available from online supplementary appendix 1. We used the Preferred Reporting Items for Systematic Reviews and Meta-Analyses flow diagram to summarise the process of study identification and inclusion. ${ }^{11}$

\section{Search strategy and selection criteria}

We searched PubMed, PsycINFO, CINAHL, EMBASE databases from inception to identify relevant studies published in the English language. Key terms for literature search included: 'need' or 'needs', 'stroke', 'survivor' and 'rehabilitation' (see search strategies in online supplementary appendix 1). The search of electronic databases was conducted on 31 March 2018. In addition, references of retrieved studies and review articles were also examined to identify relevant studies.

We included studies that met all of the following criteria: (1) reported unmet needs perceived by stroke survivors after discharge from hospital; (2) used a questionnaire or survey instrument to gather information, although studies with mixed design (both quantitative and qualitative) were included if the survey results were reported and (3) published in the English language. A study was excluded if it met any of the following exclusion criteria: (1) did not focus on stroke survivors or only considered unmet needs of carers; (2) evaluated unmet needs only before hospital discharge; (3) evaluated quality of life, patient satisfaction, or physical functions or disability, but not 'unmet needs' perceived by stroke survivors; (4) was a qualitative study, without quantitative component or (5) published in languages other than English.

Titles and abstracts of records identified by searching electronic databased were initially assessed for eligibility by two independent reviewers. The full-text articles of potentially eligible studies were collected, and examined for inclusion or exclusion by two independent reviewers. Disagreements between the reviewers were resolved by consensus.
Data extraction, quality assessment and evidence synthesis Using a pilot-tested data extraction form (online supplementary appendix 1), we gathered the following information from included studies: characteristics of stroke survivors, psychometric properties of tools used, definition of unmet needs, unmet needs perceived by stroke survivors and factors associated with perceived unmet needs. Data from multiple publications of the same study were extracted to a single data extraction form and considered together as a single study.

We assessed quality of studies based on a checklist developed by Bennett et $a l^{12}$ (online supplementary appendix $1)$. The quality assessment comprised items regarding psychometric properties of survey instruments used, sample selection and response rate.

We summarised information extracted from included studies in tables, and narratively described the main characteristics, methodological quality and study results. Reported unmet needs were categorised under three meta-themes: body functional needs, activity/participatory needs and environmental needs, ${ }^{9}$ according to the International Classification of Functioning, Disability and Health (ICF) Core Sets for Stroke framework. ${ }^{13}$ Proportions with $95 \%$ CIs of unmet needs were calculated using the Freeman-Tukey transformation methods. ${ }^{14}$ The advantage of using the Freeman-Tukey transformation is to ensure that the estimated proportions and their $95 \%$ CI are never less than $0 \%$ or larger than $100 \%$. However, quantitative meta-analyses were not conducted because of considerable heterogeneity and diversity across included studies. We reported the range of prevalence rates of a specific unmet need, where there were two or more estimates from included studies. A median of the reported rates of an unmet need was also estimated if there were three or more estimates from included studies.

\section{Patient and public involvement}

No patients and the public were involved in this systematic review.

\section{RESULTS}

Literature search initially identified a total of 2885 records. After an assessment of titles and abstracts, we examined 99 full-text articles for eligibility, and excluded 74 articles for the following reasons: not unmet needs, qualitative research only, informal caregivers only and acute stage before hospital discharge. We eventually included a total of 25 articles, corresponding to 19 eligible studies ${ }^{6-8} 15-30$ (figure 1).

The main characteristics of the included studies are shown in online supplementary appendix 2 . Two of the included studies focused on the assessment of validity and reliability of the LUNS tool, ${ }^{730}$ and one study evaluated the feasibility of the Greater Manchester Stroke Assessment Tool (GMSAT) for assessing unmet needs at 6 months after hospital discharge. ${ }^{8}$ Data from 18 included studies were analysed as cross-sectional, and only one 


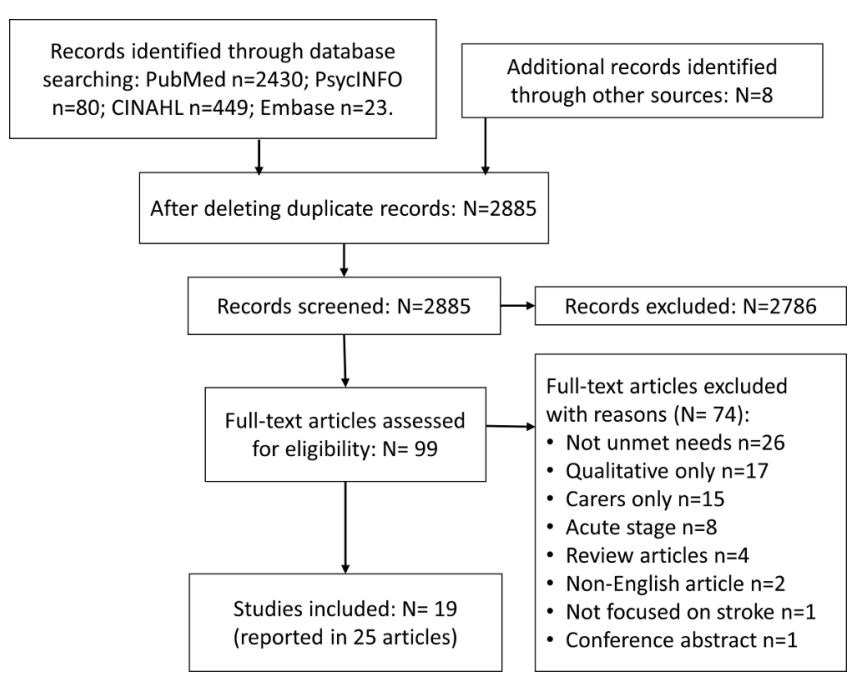

Figure 1 Preferred Reporting Items for Systematic Reviews and Meta-Analyses flow diagram for study identification and selection.

study provided results at different follow-up time points. The included studies recruited stroke survivors during 1991-2013, and the sample sizes ranged from 20 to 37383. Mean age of stroke survivors ranged from 52 to 78 years, and the proportion of men ranged from $35 \%$ to $67 \%$. Time since stroke or hospital discharge was from 1 month to 19 years.

\section{Quality of included studies}

Results of quality assessment of the included studies are presented in online supplementary appendix 3 . Out of the 19 studies, only five published full questionnaires used, seven described the core questions and seven studies did not describe details on survey questions. Out of the 19 studies, seven did not provide any information on tools' validity or reliability, and only five studies considered both validity and reliability. Acceptability and feasibility of the tools used were mentioned in six studies. Seven studies explicitly discussed how representative the study sample was, and only four studies considered sample size. Response rates ranged from $23 \%$ to $100 \%$, and it was not reported in four studies.

\section{Survey instruments used in included studies}

Tools used to measure long term unmet needs of stroke survivors are summarised in online supplementary appendix 4 . Three tools were developed specifically for measuring unmet needs perceived by stroke survivors: self-reported long-term needs after stroke (SRLNS), ${ }^{6}$ the LUNS, ${ }^{7}$ and GMSAT. $^{8}$ In terms of domains covered, the original SRLNS tool included much fewer items regarding environmental needs ${ }^{6}$ compared with the LUNS and the GMSAT tool. Psychometric properties of the SRLNS and the LUNS tool were formally evaluated in the included studies, indicating their moderate reliability, and content/concurrent validity. The validity and reliability of the GMSAT tool have not been formally evaluated, although it was considered to be feasible and acceptable. ${ }^{8}$ The completion time was about $6 \mathrm{~min}$ (range 2-12) for the LUNS tool, ${ }^{7}$ around $20 \mathrm{~min}$ for the SRLNS tool, ${ }^{24}$ and as long as $74 \mathrm{~min}$ (range 20-195) for the GMSAT tool. ${ }^{8}$ It should be noticed that time spent on a patient in the GMSAT study ${ }^{8}$ included consideration of delivering support or care services for the identified unmet needs. ${ }^{6}$ In addition to the stroke-specific tools, several studies adopted other less specific tools, including the Southampton Needs Assessment Questionnaire for people with Disability (SNAQ) ${ }^{19}$ the Impact on Participation and Autonomy Questionnaire ${ }^{21}$ and the ICF. ${ }^{23}$

\section{Unmet needs perceived by stroke survivors}

Each stroke survivor on average had two to five unmet needs, according to data from nine included studies (table 1). The proportion of stroke survivors with any unmet needs was reported in 11 included studies, with a median prevalence of $73.8 \%$ (range $19.8 \%-91.7 \%$ ). The heterogeneity in the prevalence of unmet needs across studies was likely caused by differences in patient characteristics, tools used and definitions of unmet needs. For example, a cohort study found that the prevalence of unmet needs was higher at 6 months posthospital discharge (31.5\%) than after 5years (19.8\%). ${ }^{17}$ Using the SRLNS tool, the prevalence of unmet needs was $48.9 \%$ in a study in which the unmet needs were defined as needs not met at all, ${ }^{6}$ while it was from $77.7 \%$ to $86.6 \%$ in other studies where unmet needs also included needs that were only met to some extent. ${ }^{24} 2729$ The highest prevalence rate of unmet needs $(91.7 \%)$ was found in a study that used the GMSAT tool to assess unfulfilled needs among stroke survivors at 6 months after hospital discharge and to deliver relevant care services. ${ }^{8}$ Two studies using the SNAQs tool found that $67.7 \%$ and $85.1 \%$ of younger stroke survivors (aged 21-65) had one or more unmet needs. ${ }^{19} 20$

Results of different types of unmet needs are summarised in table 2 (see online supplementary appendix 5 for results of individual studies). Fifty-five items on unmet body functioning needs were reported in nine studies, 46 items for unmet activity/participatory needs were reported in 12 studies and 102 items about environmental needs were reported in 18 studies. Estimated prevalence of unmet needs was from $59.7 \%$ to $83.7 \%$ for body functioning, and $48.4 \%$ for activities/participation. The common unmet body functioning needs included fatigue $(34.4 \%-75.1 \%)$, cognitive needs $(22.1 \%-78.4 \%)$, emotional needs $(3.3 \%-$ $72.8 \%)$ and pain $(9.1 \%-54.2 \%)$. In terms of activity and participation, the common unmet needs were related with secondary prevention $(22.1 \%-70.8 \%)$, leisure/hobbies $(8.3 \%-64.4 \%)$, mobility $(6.3 \%-46.0 \%)$ and employment/paid work $(6.9 \%-59.6 \%)$. For environmental needs, unmet service needs were the most commonly reported (table 2), including unmet information needs (3.1\%-65.0\%), transport (5.4\%-53.0\%), home help/ personal care $(4.7 \%-39.3 \%)$ and therapy $(2.0 \%-35.7 \%)$. The prevalence rate was $51.9 \%$ for unmet support needs, and it was from $1.9 \%$ to $37.6 \%$ for unmet financial/benefits needs. Other common unmet environmental needs 
Table 1 Estimated proportions of stroke survivors with one or more unmet needs

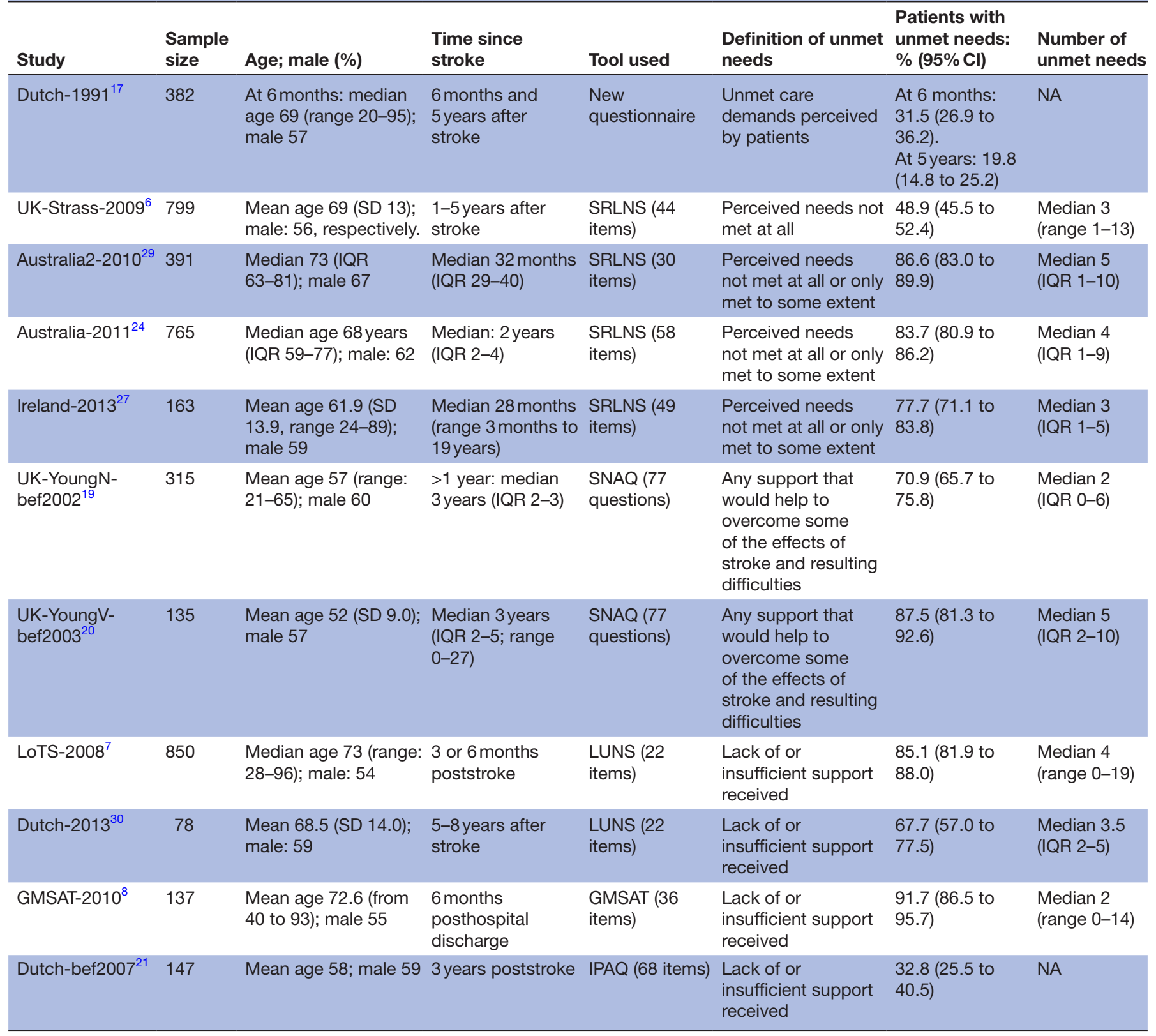

Year of starting participant recruitment was reflected in study titles in column 1; abbreviation 'bef' refers 'before' if the exact beginning year was unclear.

GMSAT, Greater Manchester Stroke Assessment Tool; IPAQ, Impact on Participation and Autonomy Questionnaire; LUNS, longer-term unmet needs after stroke tool, NA, not available, SNAQ, Southampton Needs Assessment Questionnaire; SRLNS, self-reported long-term needs after stroke.

included falls $(22.1 \%-46.0 \%)$, accessibility $(11.8 \%-$ $18.4 \%)$ and adaptation $(0.7 \%-19.2 \%)$.

Perceived unmet needs after stroke were associated with many factors, including demographic, psychological, socioeconomic characteristics and severity of disability (online supplementary appendix 6). Studies in the UK found that younger patients (aged 18-45) had more unmet needs for intellectual fulfilment, holiday and family support, than older stroke survivors (aged 46-65). ${ }^{19} 20$ According to a cohort study, the proportion of patients with any unmet needs was $31.5 \%$ at 6 months after hospital discharge and $19.8 \%$ after 5 years. ${ }^{17}$ This cohort study also reported that many participants with unmet needs at 5 years did not report any at 6 months. Compared with patients who were discharged from hospital more recently, stroke survivors after 5 years had more unmet needs for social care support and unmet needs for holidays. ${ }^{20} \mathrm{~A}$ study reported that a higher level of education was associated with more unmet needs for information. ${ }^{16}$

\section{DISCUSSION}

Existing evidence shows that postacute stroke survivors have a variety of unmet needs. Each stroke survivor on 
Table 2 Summary prevalence of specific types of unmet needs after stroke

\section{Unmet needs category/type}

Any unmet needs

Body function needs (data from nine studies):

Any unmet body function needs
Psychosocial function
Psychosocial (any)
Emotional/mood
Anxiety/depression
Personality change
Sleep pattern

Physical function

Pain

Bladder/bowel-continence

Fatigue

Sight/vision

Speech

Swallowing

Arm

Hearing

Seizures

Cognitive function:

Cognition, any

Memory/attention/concentration

Activity or participatory needs (data from 12 studies)

Any unmet activity/participation needs

Healthy lifestyle

Diet/nutritional

Holiday

Leisure/hobbies

Reading

Intellectual fulfilment

Secondary prevention

Social life

Exercise

Alcohol

Smoking

Sexual health

Physical activities

Mobility

Daily occupation/living

Walking

Writing

Independence

Employment/paid work

Driving

Speech and talk

\section{Number of items}

11

2

12

$1 \quad 8.4$

7

2

1

1

30

6

4

4

3

3

1

1

1

10

1

9

24

6

3

3

3

2

2

2

1

1

1

1

1

1

14

7

5

5

1

1

6

4

2

1

8.4

8.3

8.4

6.2

0.2

5.4

7.6

3.3

9.8
Prevalence (\%): median (range, \%) ${ }^{*}$

73.8 (19.8-91.7)

(59.7-83.7)

$39.0(3.3-72.8)$

(14.9-19.2)

$19.4(9.1-54.2)$

$19.3(9.8-51.8)$

$47.4(34.4-75.1)$

$26.6(6.2-64.4)$

$32.1(27.9-58.4)$

$31.2(5.4-43.7)$

39.1

74.6

$45.0(22.1-78.4)$

48.4

$9.3(4.7-20.9)$

$28.1(12.5-37.3)$

$22.1(8.3-64.4)$

$40.1(33.9-69.3)$

(17.5-33.7)

(22.1-70.8)

15.6

13.4

$20.7(6.3-46.0)$

19.3 (10.8-33.6)

19.6

48.0

17.1 (6.9-59.6)

(9.8-19.9)

Continued 
Table 2 Continued

\begin{tabular}{|c|c|c|}
\hline Unmet needs category/type & Number of items & Prevalence (\%): median (range, $\%)^{*}$ \\
\hline \multicolumn{3}{|c|}{ Environmental needs (data from 18 studies) } \\
\hline Support & 20 & \\
\hline Any support (domain) & 1 & 51.9 \\
\hline Financial/benefits & 10 & $17.8(1.9-37.6)$ \\
\hline Family support/role & 3 & $15.2(11.6-28.5)$ \\
\hline Relationships & 3 & $7.2(4.4-11.4)$ \\
\hline Social cultural care & 2 & $(3.6-6.9)$ \\
\hline Social support & 1 & 21.4 \\
\hline Services & 61 & \\
\hline Postacute care & 1 & 49.4 \\
\hline Information & 15 & $22.9(3.1-65.0)$ \\
\hline Therapy (any) & 13 & $13.6(2.0-35.7)$ \\
\hline Home help & 6 & $14.0(4.7-39.3)$ \\
\hline Personal care & 6 & $10.8(5.1-17.4)$ \\
\hline Transport/vehicles & 4 & $16.5(5.4-53.0)$ \\
\hline Day care & 2 & $(2.5-3.1 \%)$ \\
\hline House moving & 2 & $(7.6-10.2)$ \\
\hline Equipment maintenance & 2 & $(3.1-6.1)$ \\
\hline Respite/short breaks & 2 & $(9.0-13.5)$ \\
\hline Wheelchair & 2 & $(3.7-5.1)$ \\
\hline Continence advisor & 1 & 2.0 \\
\hline Social work & 1 & 10.1 \\
\hline Future care arrangement & 1 & 6.1 \\
\hline Mental care & 1 & 3.7 \\
\hline Weight management & 1 & 6.2 \\
\hline Body adaptation aids & 1 & 1.2 \\
\hline Safety & 11 & \\
\hline Safety (any) & 1 & 11.8 \\
\hline Falls & 6 & $28.1(22.1-46.0)$ \\
\hline Medication related & 4 & $15.2(3.3-17.1)$ \\
\hline Accessibility/accommodation & 10 & \\
\hline Accessibility (any) & 2 & $(11.8-18.4)$ \\
\hline Adaptation (any) & 8 & $9.8(0.7-19.2)$ \\
\hline
\end{tabular}

*Median (range) if there were three or more items (range) only if there were two studies. Unmet needs were categorised according to the method used by Krishnan et al. ${ }^{9}$

average had two to five unmet needs, with considerable heterogeneity in types and frequencies, depending on demographic characteristics, disability severity, years since stroke onset, health and social care services, and other environmental factors.

Except for a few studies that focused on specific types of unmet needs, most included studies considered a wide range of multidimensional unmet needs. Based on the ICF framework, ${ }^{13}$ we categorised the unmet needs after stroke into three meta-themes: body functioning, activities/participation and environmental needs. ${ }^{9}$ The included studies reported a variety of unmet needs after stroke under each of the three meta-themes, including 55 records of unmet body functioning needs in nine studies, 47 records of unmet activities/participation needs in 12 studies and 101 records of unmet environmental needs in 18 studies. Unmet body functioning needs included mainly psychosocial or cognitive problems, fatigue and pain, while unmet needs regarding activities/participation concerned mainly mobility, leisure time and employment. In terms of unmet environmental needs, the most commonly reported was the unmet needs for services, 
followed by support, accessibility/accommodation and safety. Specifically, unmet information needs and therapy needs were the most and second common of unmet needs for services.

The quality of the included survey studies was moderate or low in general. The included studies were generally satisfactory for reporting of study objectives, providing references of adopted tools and considering the strengths and limitations of a study. However, there were some quality problems, including insufficient information on the questionnaires used, inadequate information on psychometric properties of survey instruments, low response rates, missing data handling, unclear differences between responders and non-responders, and insufficient consideration of the sample's representativeness. Biases may be introduced in studies with poor quality, resulting in an overestimate or underestimate of unmet needs after stroke.

The instruments used in survey studies included those developed specifically for unmet needs among stroke survivors, ${ }^{6-8}$ and generic tools for disabled patients with any health conditions. Two of the three specific tools were formally assessed regarding psychometric properties. ${ }^{6} 7$ In terms of domains covered, the original SRLNS tool ${ }^{6}$ focused mainly on body functioning, and activity/participatory needs, although a revised version used in an Australian study contained much more items relevant to unmet environmental needs (online supplementary appendix 5) ${ }^{24}$ The three tools were all feasible and acceptable by patients and their informal carers. Compared with other two specific tools, the LUNS instrument ${ }^{7}$ covered relevant domains more comprehensively, had been more appropriately assessed for validity and reliability, and needed less time to complete (online supplementary appendix 4). The LUNS tool took a median of only $6 \mathrm{~min}$ to complete, which is an important advantage as fatigue and poor concentration are common among stroke survivors.

\section{Practice and policy implications}

Considerable heterogeneity in results of unmet needs assessment indicated that different patients, or same patients at different stages, have different specific needs for care and services. Therefore, it is reasonable to regularly assess unmet needs after stroke for making decisions on the development and provision of health and social care services. The guideline on stroke rehabilitation by the National Institute for Health and Care Excellence recommended that the health and social care needs of stroke survivors should be assessed at 6 months posthospital discharge and then annually thereafter. ${ }^{31}$ However, there remains very limited evidence on the clinical implementation of the assessment of LUNS. The GMSAT tool has been designed to directly address the identified unmet needs at 6 months after hospital discharge, in which 50.4\% of unmet needs could be addressed by providing information/advice, $19.8 \%$ were addressed by signposting to the community services and $21.1 \%$ to general practices. ${ }^{8}$ However, limited evidence from randomised controlled trials failed to demonstrate the effects of structured assessment of longer term problems among stroke survivors at 6 months after hospital discharge. ${ }^{32}{ }^{33}$ It remains unclear whether the identification of unmet needs will result in the improvement of patients' activity, participation and quality of life.

\section{Limitations}

There are several limitations in the present systematic review. First, we focused on 'unmet needs' and excluded studies that assessed needs after stroke but did not explicitly report results regarding 'unmet needs'. Second, the present systematic review included studies of stroke survivors, and excluded studies of only informal carers. Third, the included studies were from high-income developed countries, so that results may not be generalisable to low-and-middle-income countries due to different health and social care systems. Finally, the results of the included studies might be biased due to some methodological problems, such as participant recruitment and response bias. Results of different studies may not be directly comparable due to different tools used, varying domains and different definitions of unmet needs after stroke.

\section{Further research required}

This systematic review included only one study that evaluated unmet needs at different time points, so that further longitudinal studies are required to evaluate changes in unmet needs of stroke survivors over time. Because of different tools used, there was often a lack of comparability of evidence generated from different studies on the topic. Validated tools (such as SRLNS ${ }^{64}$ or LUNS ${ }^{730}$ should be used in future studies of unmet needs of postacute stroke survivors. WHO ICF framework seems the most comprehensive and promising model, which is not only useful for assessing unmet needs of stroke survivors, but also potentially helpful for the comparison of unmet needs across different conditions and for patients with multimorbidity conditions. The included studies reported a large number of poststroke unmet needs under different domains or subthemes, and further research is required to understand the complex interaction or interconnectedness of unmet needs across domains. The ultimate purpose of the unmet needs assessment is to improve patient outcomes in terms of body functioning, activities, participation and quality of life. There is very limited evidence from relevant clinical trials, ${ }^{32-34}$ and more research is required to link identified unmet needs with the development of health and social care policies and practice guidelines, and the provision of cost-effective interventions. Finally, available evidence on unmet needs after stroke was mainly from high-income countries, and more studies in low-and-middle-income countries are required to generate locally relevant evidence on multidimensional needs of stroke survivors. 


\section{CONCLUSIONS}

The estimated prevalence of postacute unmet needs after stroke was high, and there was considerable heterogeneity in type and frequency of specific unmet needs. Further research is required to link regular assessment of long term unmet needs of stroke survivors with the provision of cost-effective patient-centred health and social care services.

Contributors FS, TC and LZ developed the review protocol. FS conducted literature search. FS, TC, LZ, BZ and YD contributed to eligibility assessment and study inclusion. FS, TC, BZ, YD and J-CF extracted data from included studies. FS analysed data and drafted and all authors commented on the manuscript.

Funding The authors have not declared a specific grant for this research from any funding agency in the public, commercial or not-for-profit sectors.

Competing interests None declared.

Patient consent for publication Not required.

Provenance and peer review Not commissioned; externally peer reviewed.

Data sharing statement All data relevant to the study are included in the article or uploaded as online supplementary information.

Open access This is an open access article distributed in accordance with the Creative Commons Attribution Non Commercial (CC BY-NC 4.0) license, which permits others to distribute, remix, adapt, build upon this work non-commercially, and license their derivative works on different terms, provided the original work is properly cited, appropriate credit is given, any changes made indicated, and the use is non-commercial. See: http://creativecommons.org/licenses/by-nc/4.0/.

\section{REFERENCES}

1. Feigin VL, Norrving B, Mensah GA. Global burden of stroke. Circ Res 2017;120:439-48.

2. Murray $\mathrm{J}$, Young $\mathrm{J}$, Forster A. Measuring outcomes in the longer term after a stroke. Clin Rehabil 2009;23:918-21.

3. Sumathipala K, Radcliffe E, Sadler E, et al. Identifying the long-term needs of stroke survivors using the International Classification of Functioning, Disability and Health. Chronic IIIn 2012;8:31-44.

4. Asadi-Lari M, Tamburini M, Gray D. Patients' needs, satisfaction, and health related quality of life: towards a comprehensive model. Health Qual Life Outcomes 2004:2:32.

5. Stevens A, Gillam S. Needs assessment: from theory to practice. BMJ 1998;316:1448-52.

6. McKevitt C, Fudge N, Redfern J, et al. Self-reported long-term needs after stroke. Stroke 2011;42:1398-403.

7. LoTS care LUNS study team. Validation of the longer-term unmet needs after stroke (LUNS) monitoring tool: a multicentre study. Clin Rehabil 2013;27:1020-8.

8. Rothwell K, Boaden R, Bamford D, et al. Feasibility of assessing the needs of stroke patients after six months using the GM-SAT. Clin Rehabil 2013;27:264-71.

9. Krishnan S, Pappadis MR, Weller SC, et al. Needs of stroke survivors as perceived by their caregivers: a scoping review. Am J Phys Med Rehabil 2017;96:487-505.

10. Hafsteinsdóttir TB, Vergunst M, Lindeman E, et al. Educational needs of patients with a stroke and their caregivers: a systematic review of the literature. Patient Educ Couns 2011;85:14-25.

11. Liberati A, Altman DG, Tetzlaff J, et al. The PRISMA statement for reporting systematic reviews and meta-analyses of studies that evaluate health care interventions: explanation and elaboration. $J$ Clin Epidemiol 2009;62:e1-e34.
12. Bennett C, Khangura S, Brehaut JC, et al. Reporting guidelines for survey research: an analysis of published guidance and reporting practices. PLoS Med 2010;8:e1001069.

13. Geyh S, Cieza A, Schouten J, et al. ICF Core Sets for stroke. J Rehabil Med 2004:135-41.

14. Freeman MF, Tukey JW. Transformations related to the angular and the square root. The Annals of Mathematical Statistics 1950;21:607-11.

15. Brandriet LM, Lyons M, Bentley J. Perceived needs of poststroke elders following termination of home health services. Nurs Health Care 1994;15:514-20.

16. van Veenendaal H, Grinspun DR, Adriaanse HP. Educational needs of stroke survivors and their family members, as perceived by themselves and by health professionals. Patient Educ Couns 1996:28:265-76.

17. op Reimer WJ, Scholte de Haan RJ, Rijnders PT, et al. Unmet care demands as perceived by stroke patients: deficits in health care? Qual Health Care 1999;8:30-5.

18. Zwygart-Stauffacher M, Lindquist R, Savik K. Development of health care delivery systems that are sensitive to the needs of stroke survivors and their caregivers. Nurs Adm Q 2000;24:33-42.

19. Kersten P, Low JT, Ashburn A, et al. The unmet needs of young people who have had a stroke: results of a national UK survey. Disabil Rehabil 2002;24:860-6.

20. Low JT, Kersten P, Ashburn A, et al. A study to evaluate the met and unmet needs of members belonging to Young Stroke groups affiliated with the Stroke Association. Disabil Rehabil 2003;25:1052-6.

21. van de Port IG, van den Bos GA, Voorendt M, et al. Identification of risk factors related to perceived unmet demands in patients with chronic stroke. Disabil Rehabil 2007;29:1841-6.

22. Tistad $\mathrm{M}$, Tham $\mathrm{K}$, von $\mathrm{Koch} \mathrm{L}$, et al. Unfulfilled rehabilitation needs and dissatisfaction with care 12 months after a stroke: an explorative observational study. BMC Neurol 2012;12:40.

23. Duxbury S, Depaul V, Alderson M, et al. Individuals with stroke reporting unmet need for occupational therapy following discharge from hospital. Occup Ther Health Care 2012;26:16-32.

24. Andrew NE, Kilkenny M, Naylor R, et al. Understanding longterm unmet needs in Australian survivors of stroke. Int J Stroke 2014;9:106-12.

25. Platten M. Identifying and meeting people's post-stroke emotional needs: a service evaluation. British Journal of Neuroscience Nursing 2014:10:191-7.

26. Ekstam L, Johansson U, Guidetti S, et al. The combined perceptions of people with stroke and their carers regarding rehabilitation needs 1 year after stroke: a mixed methods study. BMJ Open 2015;5:e006784.

27. Walsh ME, Galvin R, Loughnane $\mathrm{C}$, et al. Community re-integration and long-term need in the first five years after stroke: results from a national survey. Disabil Rehabil 2015;37:1834-8.

28. Ullberg T, Zia E, Petersson J, et al. Perceived unmet rehabilitation needs 1 year after stroke: an observational study from the Swedish stroke register. Stroke 2016;47:539-41.

29. Olaiya MT, Cadilhac DA, Kim J, et al. Long-term unmet needs and associated factors in stroke or TIA survivors: An observational study. Neurology 2017;89:68-75.

30. Groeneveld IF, Arwert HJ, Goossens PH, et al. The longer-term unmet needs after stroke questionnaire: cross-cultural adaptation, reliability, and concurrent validity in a Dutch population. J Stroke Cerebrovasc Dis 2018;27:267-75.

31. NICE. Stroke rehabilitation in adults (CG162). NICE Guidelines. Manchester: National Institute for Health and Care Excellence, 2013.

32. Forster A, Young J, Green J, et al. Structured re-assessment system at 6 months after a disabling stroke: a randomised controlled trial with resource use and cost study. Age Ageing 2009;38:576-83.

33. Forster A, Young J, Chapman K, et al. Cluster randomized controlled trial: clinical and cost-effectiveness of a system of longer-term stroke care. Stroke 2015;46:2212-9.

34. Forster A, Young J, Nixon J, et al. Protocol of a cluster randomized trial evaluation of a patient and carer-centered system of longer-term stroke care (LoTS care). Int J Stroke 2015;10:259-63. 